\title{
Conversion Scheme for DCT-Domain Transcoding of MPEG-2 to H.264/AVC
}

\author{
Joo-kyong Lee and Ki-dong Chung \\ Dept. of Computer Engneering, Pusan National Univ., Jangjeon-dong, \\ Geumjeong-gu, Busan, 609-735, Korea \\ jklee@melon.cs.pusan.ac.kr, kdchung@pusan.ac.kr
}

\begin{abstract}
The $4 \times 4$ approximate discrete cosine transform (DCT) of H.264/AVC [1] makes it difficult to transcode the pre-coded video contents with the previous video coding standards to H.264/AVC in DCT domain. This is due to the difference between $8 \times 8$ DCT used previous standards and $4 \times 4$ DCT in H.264/AVC. In this paper, we propose an efficient algorithm that converts the quantized $8 \times 8$ DCT block of MPEG- 2 into newly quantized four $4 \times 4$ DCT blocks of H.264/AVC to support DCT-domain transcoding. Experimental results show that the proposed scheme improves computational complexity by $5 \sim 11 \%$ and video quality by $0.1 \sim 0.5 \mathrm{~dB}$ compared with cascaded pixeldomain transcoding that exploits inverse quantization (IQ), inverse DCT (IDCT), DCT, and re-quantization (re-Q).
\end{abstract}

\section{Introduction}

As the number of networks, types of devices, and video representation formats increase, interoperability between different systems and different networks is becoming more and more important [2]. To provide a seamless interaction between producers and consumers, diverse research on video transcoding such as bit-rate reduction [3], spatial resolution reduction [4], frame skipping [5][6], and simple video format conversion [7], has been conducted. Recently, DCT-domain transcoding approaches have been studied to improve computational complexity and to avoid DCT and IDCT mismatch problem [8][9]. These approaches are based on $8 \times 8$ DCT used in most video coding standards such as MPEG-2, MPEG-4, and H.263/AVC. However, it is impossible to directly apply them to H.264/AVC, because it uses $4 \times 4$ transforms.

In this paper, for non-intra-coded blocks, we propose an efficient algorithm to convert an $8 \times 8$ DCT block of MPEG- 2 to four $4 \times 4$ DCT blocks of H.264/AVC. Additionally, we propose a quantization conversion algorithm that changes quantization step size between the two standards. This algorithm improves video quality by reducing the quantization error caused by cascaded IQ/re-Q. However, for intra-coded blocks, we follow the cascaded re-encoding method in pixel domain instead of DCT conversion to avoid heavy computational complexity in prediction mode selection. Our scheme can be easily applied to other standards by slightly modifying the quantization conversion algorithm. This paper is organized as follows. In Section 2 we de- 
scribe $4 \times 4$ transformation for H.264/AVC, respectively. In Section 3 we present the Qstep conversion scheme and DCT conversion scheme. Experimental results will be presented in Section 4, and the conclusion is shown in Section 5.

\section{Transformation for H.264/AVC [10] [11]}

The $4 \times 4$ transformation of H.264/AVC approximates the 'true' $4 \times 4$ DCT. The $4 \times 4$ transform matrix, $H$ of the 'true' DCT can be illustrated by Eq. 1, where $a, b, c$ represent the matrix elements. H.264/AVC, On the other hand, uses the modified transform matrix $H^{\prime}$, where $b$ and $b / c$ are changed to $\sqrt{2 / 5}$ and $\frac{1}{2}$, respectively. In actual H.264/AVC implementations, the integer transform matrix is used for transformation, and the residual scaling factors are absorbed into quantization process to avoid multiplication operations, and maintain DCT/IDCT accuracy. See [10] as well as [11] for more detail. In this paper we use $H^{\prime}$ to convert the $8 \times 8$ DCT block into four $4 \times 4$ DCT blocks of H.264/AVC, and we cover the residual scaling factors using quantization step size (Qstep) conversion tool. Throughout this paper, we represent the result of the modified $4 \times 4$ DCT using $H^{\prime}$ as ' $4 \times 4$ approximate DCT'. Since there is a difference between 'true' DCT and 'approximate' DCT, we should compensate it when we convert the $8 \times 8$ DCT block into four $4 \times 4$ approximate DCT blocks.

$$
Y=H X H^{T}, H=\left[\begin{array}{cccc}
a & a & a & a \\
b & c & -c & -b \\
a & -a & -a & a \\
c & -b & b & -c
\end{array}\right] \text {, where } a=\frac{1}{2}, b=\sqrt{\frac{1}{2}} \cos \left(\frac{\pi}{8}\right), c=\sqrt{\frac{1}{2}} \cos \left(\frac{3 \pi}{8}\right)
$$

\section{Proposed Conversion Schemes}

In this section we present two conversion schemes, Qstep conversion scheme and the DCT conversion scheme. The Qstep conversion scheme changes the quantized DCT coefficients of MPEG-2 to newly quantized coefficients of H.264/AVC. This scheme covers the H.264/AVC quantizer that is associated with transformation by incorporating the residual scaling factors into it. DCT conversion scheme converts the 'true' $8 \times 8$ DCT into the $4 \times 4$ approximate DCT.

\subsection{Qstep Conversion}

In MPEG-2 encoder, intra coded macroblock (MB) and non-intra coded MB are quantized by different formula and quantizer matrices. Intra coded MB coefficients are quantized as shown in Eq. 2, where $\hat{B}_{(i, j)}$ refers to the $(i, j)^{\text {th }}$ coefficient of the DCT block $\hat{B}, Q\left(\hat{B}_{(i, j)}\right)$ refers to the quantized value of $\hat{B}_{(i, j)}$, and $q$ refers to Qstep value. The DC coefficients of the luminance and chrominance components of the intra coded $\mathrm{MB}$ are divided by one of the Qstep values 8, 4, 2 and 1. For AC coefficients, first, the coefficients are scaled by 32 and corresponding elements of the intra quantizer matrix $W_{(i, j)}^{\text {intra }}$, and secondly, they are quantized by Qstep value $q$ ranging from 1 to 112 . 


$$
\begin{aligned}
Q\left(\hat{B}_{(i, j)}\right)=\left\{\begin{array}{c}
\hat{B}_{(i, j)} / q \text { for } i=j=0 \\
\left\{\left(32 \cdot \hat{B}_{(i, j)}\right)+\operatorname{sign}\left(\hat{B}_{(i, j)}\right) \cdot q \cdot W_{(i, j)}^{\text {intra }}\right\} /\left(2 q \cdot W_{(i, j)}^{\text {intra }}\right) \text { for all } i, j \text { except } i=j=0 \\
, \text { where } \quad \operatorname{sign}\left(\hat{B}_{(i, j)}\right)=\left\{\begin{array}{cc}
1, & \hat{B}_{(i, j)}>0 \\
0, & \hat{B}_{(i, j)}==0 \\
-1, & \hat{B}_{(i, j)}<0
\end{array}\right.
\end{array}\right. \\
\hat{B}_{(i, j)}=\frac{q \cdot W_{(i, j)}^{\text {intra }}}{16}\left\{Q\left(\hat{B}_{(i, j)}\right)-\frac{\operatorname{sign}\left(\hat{B}_{(i, j)}\right)}{2}\right\} \\
\frac{\hat{B}_{(i, j)}}{q^{\prime}}=\frac{q}{q^{\prime}} \cdot \frac{W_{(i, j)}^{\text {intra }}}{16} \cdot\left\{Q\left(\hat{B}_{(i, j)}\right)-\frac{\operatorname{sign}\left(\hat{B}_{(i, j)}\right)}{2}\right\} \\
Q\left(\hat{B}_{(i, j)}\right)=\left(32 \cdot \hat{B}_{(i, j)}\right) /\left(2 q \cdot W_{(i, j)}^{\text {non-intra }}\right), 0 \leq i, j \leq 7 \\
\hat{B}_{(i, j)}=\frac{q}{16} W_{(i, j)}^{n o n-\text { intra }} \cdot Q\left(\hat{B}_{(i, j)}\right) \\
\frac{\hat{B}_{(i, j)}}{q^{\prime}}=\frac{q}{q^{\prime}} \cdot \frac{W_{(i, j)}^{\text {non-intra }}}{16} \cdot Q\left(\hat{B}_{(i, j)}\right)
\end{aligned}
$$

Now let us change Eq. 2 to transcode the quantized coefficients, $Q\left(\hat{B}_{(i, j)}\right)$, to newly quantized values of H.264/AVC. First, the DC coefficients are consecutively dequantized by $q$ and then re-quantized by new Qstep $q^{\prime}$. Secondly, the unquantized AC coefficients, $\hat{B}_{(i, j)}$, of MPEG-2 as described in Eq. 3, derived from Eq. 2, can be calculated the newly quantized coefficients using $q^{\prime}$ as described in Eq. 4. Briefly, using Eq. 4, we convert quantized AC coefficients of MPEG-2 into newly quantized coefficients of H.264/AVC. This method has an effect of skipping MPEG-2 quantization. That is, we can calculate the newly quantized coefficients of H.264/AVC as if we performed quantization only once with the unquantized coefficients, $\hat{B}_{(i, j)}$ without any quantization for MPEG-2. As a result, the proposed Qstep conversion scheme can reduce the dequantization errors of MPEG-2 bit-stream compared with cascaded pixel-domain scheme.

As in the case of intra-MB conversion, Eq. 6 for unquantized coefficients is derived from the MPEG-2 quantization formula, Eq. 5, where $W_{(i, j)}^{\text {non-intra }}$ indicates $(i, j)^{\text {th }}$ element of the quantizer matrix for non-intra MB, and the newly quantized coefficients $\hat{B}_{(i, j)} / q^{\prime}$ can be calculated by dividing $\hat{B}_{(i, j)}$ by $q^{\prime}$.

\subsection{DCT Conversion}

The $8 \times 8$ DCT block conversion into four $4 \times 4$ approximate DCT blocks is extended from the pixel-domain extracting. In the pixel-domain, extracting of the $4 \times 4$ subblock $B_{i}$ from $B$ is defined by Eq. 8 , where $B$ is an $8 \times 8$ motion compensated (MC) block or a intra block in the pixel-domain, and $L_{i}, R_{i}, i=0, . ., 3$ is the displacement ma- 
trices that perform vertical and horizontal filtering, respectively. The order of subblock $B_{i}$ is a raster scan order in $B$. The matrices $L_{i}$ size of $4 \times 8$ and $R_{i}$ size of $8 \times 4$ are defined in Eq. 9, where $I_{4 \times 4}$ is identity matrix of size 4 . The pre-multiplication by $L_{i}$ vertically extracts a sub-block size of $4 \times 8$ from $B$ whereas, the post-multiplication by $R_{i}$ horizontally extracts $4 \times 4$ block from the resultant block.

$$
\begin{aligned}
& B_{i}=L_{i} \cdot B \cdot R_{i}(0 \leq i \leq 3) \\
& L_{0}=L_{1}=\left(\begin{array}{ll}
I_{4 \times 4} & 0
\end{array}\right)_{4 \times 8}, \quad L_{2}=L_{3}=\left(\begin{array}{ll}
0 & I_{4 \times 4}
\end{array}\right)_{4 \times 8}, \\
& R_{0}=R_{2}=\left(\begin{array}{c}
I_{4 \times 4} \\
0
\end{array}\right)_{8 \times 4}, R_{1}=R_{3}=\left(\begin{array}{c}
0 \\
I_{4 \times 4}
\end{array}\right)_{8 \times 4}
\end{aligned}
$$

The $4 \times 4$ DCT block can be calculated by performing DCT on the extracted sub-block, $B_{i}$ of Eq. 8. Because of the distributive property of matrix multiplication with respect to the DCT, transformed matrix of $B_{i}$ is given by $\hat{B}_{i}=\hat{L}_{i} \cdot \hat{B} \cdot \hat{R}_{i}$ as represented in Eq. 10, where $\hat{B}_{i}, \hat{L}_{i}, \hat{B}$, and $\hat{R}_{i}$ are the DCT representations of $B_{i}, L_{i}, B$ and $R_{i}$ respectively. Especially, $\hat{L}_{i}$ and $\hat{R}_{i}$ can be written in the form of $H_{4 \times 4} \cdot L_{i} \cdot H_{8 \times 8}^{T}$ and $H_{8 \times 8} \cdot R_{i} \cdot H_{4 \times 4}^{T}$ as described in Eq. 11, where $H_{4 \times 4}$ and $H_{8 \times 8}$ are the transform matrices for $4 \times 4$ and $8 \times 8$ DCT, respectively, and $H_{4 \times 4}^{T}$ indicates the transpose of $H_{4 \times 4}$. Consequently, $\hat{B}_{i}$ can be rewritten as the form of Eq. 11. Through this equation, we can simply change an $8 \times 8$ DCT block into four $4 \times 4$ DCT blocks, because the transform matrices, $H_{4 \times 4}, H_{4 \times 4}^{T}, H_{8 \times 8}, H_{8 \times 8}^{T}$, and filtering matrices $L_{i}, R_{i}(0 \leq i \leq 3)$ are already known matrices and we can make up the look-up table using them. However, $\hat{B}_{i}(0 \leq i \leq 3)$ is not identical with the $4 \times 4$ approximate DCT block, $\tilde{B}_{i}$ of H.264/AVC, because the transform matrices are different as mentioned in section 2. Accordingly, it is necessary to compensate the difference, and Eq. 12 shows our final formula. In Eq. 12, we can obtain $\tilde{B}_{i}$ by using $H_{4 \times 4}^{\prime}$, which changes elements of $H_{4 \times 4}$, $b=\sqrt{1 / 2} \cos \left(\frac{\pi}{8}\right) \approx 0.6533$ and $c / b=0.414$ to $b=\sqrt{2 / 5}$ and $c / b=1 / 2$, instead of $H_{4 \times 4}$. The specific derivation processes are shown in Eq. $13 \sim$ Eq. 15. In Eq. 14, $H_{8 \times 8}{ }^{T} H_{8 \times 8}$ and $H_{8 \times 8} H_{8 \times 8}{ }^{T}$ are an identity matrix, respectively, due to the orthogonal property of the DCT transform matrix. Lastly, we can get the form of Eq. 15 by applying the associative property of matrix multiplication to Eq. 14. This Equation is equal to Eq. 12, because the matrix multiplication $H_{8 \times 8} \cdot B \cdot H_{8 \times 8}^{T}$ is identical to $\hat{B}$. As a consequence, we can exactly converts an $8 \times 8$ DCT block into four $4 \times 4$ approximate DCT blocks of H.264/AVC with the modified transform matrix $H_{4 \times 4}^{\prime}$. As stated earlier, the product of matrices, $H_{4 \times 4}^{\prime} \cdot L_{i} \cdot H_{8 \times 8}^{T}$ and $H_{8 \times 8} \cdot R_{i} \cdot H_{4 \times 4}^{\prime T}$ in Eq. 12 can be pre-computed and stored in the memory as a look-up table.

$$
\begin{aligned}
\operatorname{DCT}\left(B_{i}\right) & =D C T\left(L_{i} \cdot B \cdot R_{i}\right)=D C T\left(L_{i}\right) \cdot \operatorname{DCT}(B) \cdot D C T\left(R_{i}\right) \\
& =\hat{B}_{i}=\hat{L}_{i} \cdot \hat{B} \cdot \hat{R}_{i}, \quad(0 \leq i \leq 3)
\end{aligned}
$$




$$
\begin{aligned}
\hat{B}_{i} & =\left(H_{4 \times 4} \cdot L_{i} \cdot H_{8 \times 8}^{T}\right) \cdot(\hat{B}) \cdot\left(H_{8 \times 8} \cdot R_{i} \cdot H_{4 \times 4}^{T}\right)(0 \leq i \leq 3) \\
\tilde{B}_{i} & =\left(H_{4 \times 4}^{\prime} \cdot L_{i} \cdot H_{8 \times 8}^{T}\right) \cdot(\hat{B}) \cdot\left(H_{8 \times 8} \cdot R_{i} \cdot H_{4 \times 4}^{\prime T}\right)(0 \leq i \leq 3) \\
\tilde{B}_{i} & =H_{4 \times 4}^{\prime} \cdot B_{i} \cdot H_{4 \times 4}^{\prime T}=H_{4 \times 4}^{\prime} \cdot\left(L_{i} \cdot B \cdot R_{i}\right) \cdot H_{4 \times 4}^{\prime T}=\left(H_{4 \times 4}^{\prime} \cdot L_{i}\right) \cdot B \cdot\left(R_{i} \cdot H_{4 \times 4}^{\prime T}\right) \\
& =\left(H_{4 \times 4}^{\prime} \cdot L_{i}\right) \cdot\left(H_{8 \times 8}^{T} \cdot H_{8 \times 8}\right) \cdot B \cdot\left(H_{8 \times 8}^{T} \cdot H_{8 \times 8}\right) \cdot\left(R_{i} \cdot H_{4 \times 4}^{\prime T}\right) \\
& =\left(H_{4 \times 4}^{\prime} \cdot L_{i} \cdot H_{8 \times 8}^{T}\right) \cdot\left(H_{8 \times 8} \cdot B \cdot H_{8 \times 8}^{T}\right) \cdot\left(H_{8 \times 8} \cdot R_{i} \cdot H_{4 \times 4}^{\prime T}\right)
\end{aligned}
$$

\section{Experimental Results}

Throughout the various experiments we compare the performance of our proposed scheme with the cascaded re-encoding method of pixel-domain. In this paper, we call the cascaded re-encoding method "cascade" method. For simulation, we implemented DCT-based transcoding architecture and cascaded pixel-based transcoding architecture by modifying the MPEG-2 Test Model 5 (TM5) codec [12] and adopting the H.264 Joint Model 8 (JM8) encoder partially [13]. For convenient simulation, we fixed Qstep for all frames in a video sequence. In experiments, we tested three video sequences with the different motion characteristic: FOOTBALL (high motion degree), CARPHONE (medium degree), and CLAIRE (low degree). In the simulation, input video sequences of Quarter Common Intermediate Format (QCIF) were encoded by the MPEG-2 encoder at constant frame rate of 30, GOP size of 6 , and I/P distance of 3, and then, the output bit-streams of the MPEG-2 encoder are transcoded to H.264 bit-streams.

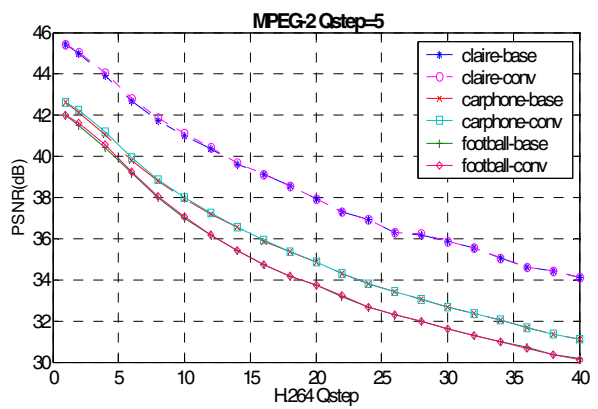

Fig. 1. The comparison of PSNR for test video sequences when Qstep is fixed to 5 in MPEG-2 encoding and the re-Qstep is changed from 1 to 40

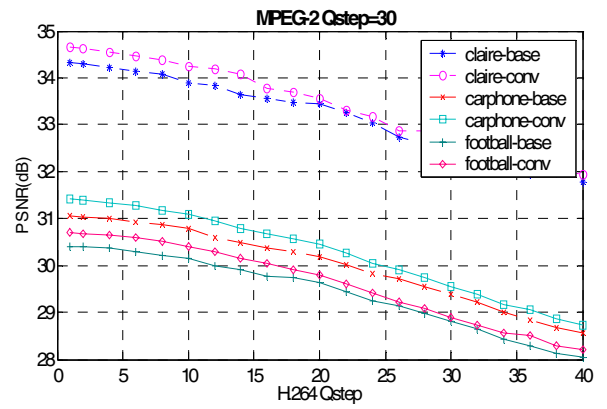

Fig. 2. The comparison of PSNR for test video sequences when Qstep is fixed to 30 in MPEG-2 encoding and the re-Qstep is changed from 1 to 40

Fig. 1 and Fig. 2 shows the peak signal-to-noise ratio (PSNR) comparison as changing the re-Qstep from 1 to 40 of the transcoder at fixed MPEG-2 Qstep 5 and 30, respectively. In the legend of the figures, '-base' refers to cascade method and '-conv' refers to our proposed scheme. In Fig. 1, we cannot make a discrimination between the 
performance of the proposed scheme and the cascade method even though our method is numerically higher than the cascade method by $0.01 \sim 0.04 \mathrm{~dB}$. Contrast to this result, Fig. 2 shows different results that PSNR of our method is higher than the cascade method by maximum $0.45 \mathrm{~dB}$. This is due to our quantization scheme that has an effect of skipping MPEG-2 quantization as explained in section 3.1.

Fig. 3 and Fig. 4 show PSNR of the frames ranging from 1 to 30 for CARPHONE and CLAIRE sequences, respectively. With this figures, we can observe that, as a whole, the proposed scheme shows higher performance than the cascade method. Especially, for I frames, it is more superior to the cascade method. The reason is due to the magnitude of the Motion Compensated DCT (MC-DCT) coefficients. More specifically, because the magnitude of the intra-coded block coefficients is generally larger than the inter-coded block coefficients, the quantization errors of the intracoded block are larger than the inter-coded block at high Qstep. Conversely, because our scheme has an effect of skipping the MPEG-2 quantization process, it can maintain the video quality.

Fig. 5 shows the reconstructed real images carried out the transcoding process at Qstep 20 for MPEG-2 encoding and at re-Qstep 6 for H.264/AVC transcoding. The cascade method images are arranged in the first row, while the proposed method images are in the second row. FOOTBALL, CARPHONE, and CLAIRE images are arranged from left to right. Let us look hard at the images and compare each pairs. First, for the FOOTBALL sequence, the second player's hip area of our image (b) is clearer than that of the cascade method (a). Second, for CARPHONE, we cannot discover notable difference. Third, for CLAIRE, the woman's silhouette and background of our image (f) are clearer without blurring than the cascade method (e).

Finally, we represent the computational complexity by comparing the number of multiplications and additions. We have implemented IQ, IDCT, DCT, and re-Q for the cascade method, and quantization conversion and DCT conversion for the proposed method, respectively. For the cascade method, we do not consider fast DCT/IDCT methods, but only consider the conventional DCT/IDCT methods. Fast DCT/IDCT methods can reduce computational complexity by re-using intermediately calculated values instead of several multiplications and additions for transform. Even though our proposed method also can be implemented by the same kind of fast

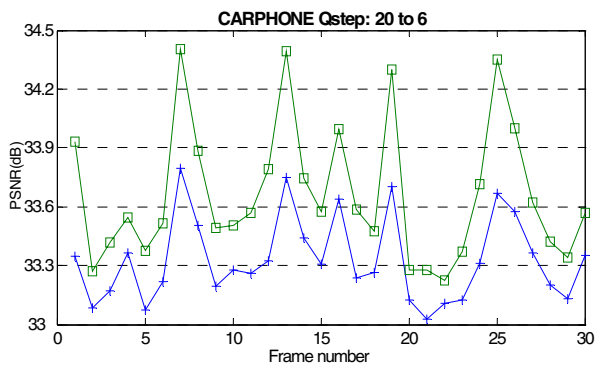

Fig. 3. The PSNR performance for the CARPHONE sequence ranging from frame 1 to frame 30, where Qstep is set to 20 for MPEG-2 encoding and re-Qstep is set to 6 for H.264/AVC

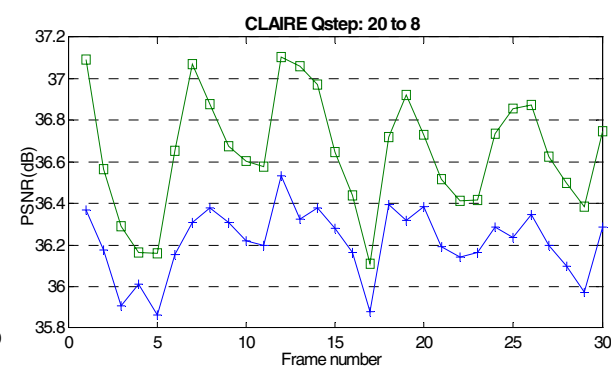

Fig. 4. The PSNR performance for the CLAIRE sequence ranging from frame 1 to frame 30, where Qstep is set to 20 for MPEG2 encoding and re-Qstep is set to 8 for H.264/AVC 


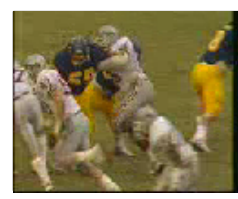

(a)

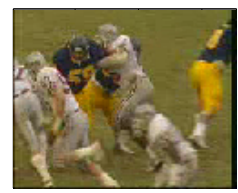

(b)

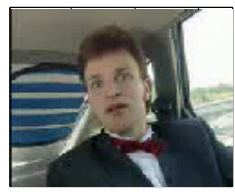

(c)

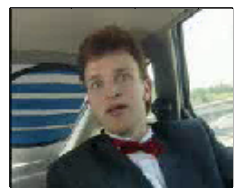

(d)

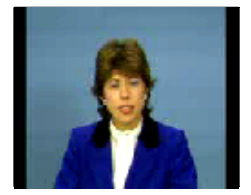

(e)

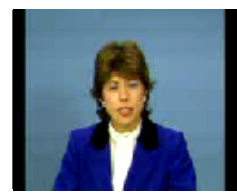

(f)

Fig. 5. The real images for the sample video sequences at $\mathrm{Q} s t e p=20$ and re-Qstep=6: FOOTBALL (a)(b) CARPHONE (c)(d) CLAIRE (e)(f). The cascade method images are arranged in the first row, while the proposed method images are in the second row.

Table 1. The required operations to transcode a quantized $8 \times 8$ DCT block to newly quantized $4 \times 4$ approximate DCT blocks

\begin{tabular}{c|c|c|c}
\hline Function & $\begin{array}{c}\text { Cascade method } \\
\text { (Inter or Intra block) }\end{array}$ & $\begin{array}{c}\text { Proposed method } \\
\text { (Inter) }\end{array}$ & $\begin{array}{c}\text { Proposed method } \\
\text { (Intra) }\end{array}$ \\
\hline $\begin{array}{c}\text { IQ/Q } \\
\text { (or Qcon) }\end{array}$ & $192 \mathrm{M}+128 \mathrm{~A}+192 \mathrm{~S}$ & $64 \mathrm{M}+1 \mathrm{D}$ & $\begin{array}{c}128 \mathrm{M}+1 \mathrm{D}+64 \mathrm{~A}+64 \\
\mathrm{~S}\end{array}$ \\
\hline DCT conversion & - & $1,024 \mathrm{M}+896 \mathrm{~A}$ & - \\
\hline $8 \times 8$ IDCT & $1,024 \mathrm{M}+896 \mathrm{~A}$ & - & $1024 \mathrm{M}+896 \mathrm{~A}$ \\
\hline $4 \times 4$ approximate DCT & $256 \mathrm{~A}+64 \mathrm{~S}$ & - & $256 \mathrm{~A}+64 \mathrm{~S}$ \\
\hline \hline Total operations & $1,216 \mathrm{M}+1,280 \mathrm{~A}+256 \mathrm{~S}$ & $1,088 \mathrm{M}+1 \mathrm{D}+896 \mathrm{~A}$ & $\begin{array}{c}1,152 \mathrm{M}+1 \mathrm{D}+1,024 \\
\mathrm{~A}+128 \mathrm{~S}\end{array}$ \\
\hline
\end{tabular}

method, we do not consider the fast method in this paper. For intra-coded block, we use the cascaded re-encoding method in pixel domain to avoid too much computation complexity.

Table 1 shows the number of operations to transcode a quantized block of $8 \times 8$ size of MPEG-2 to newly quantized four $4 \times 4$ approximate DCT blocks of H.264/AVC, where "M" stands for multiplication operations, "A" for adds, "S" for Shift, and "D" for division. For a brief comparison of computational complexity, we ignore the number of addition, shift, and subtraction operations because the overhead of the multiplication and division operations are higher than any other operation. According to Table 1, the proposed scheme allows a computational complexity saving of about $11 \%$ for non-intra-coded blocks and about $5 \%$ for intra-coded blocks, compared with the cascade method. 


\section{Conclusions}

In this paper, we proposed an efficient and exact conversion algorithm for the quantized $8 \times 8$ DCT block of MPEG- 2 into newly quantized four $4 \times 4$ approximate DCT blocks of H.264/AVC to support DCT-domain transcoding. With the slight modification of quantization conversion, this method also can be applied to MPEG-1, MPEG-4 and H.263, etc. Extensive simulation results show that the PSNR of the proposed method outperforms the cascaded re-recoding method in the pixel domain by $0.1 \sim$ $0.5 \mathrm{~dB}$ and the computational complexity can be reduced by $5 \sim 11 \%$. Our next subject is to find out an efficient half pixel conversion algorithm to compensate the difference of half pixel value between MPEG-2 and H.264/AVC and is to integrate the algorithm and our DCT conversion algorithm.

\section{References}

1. ISO/IEC 14496-10:2003, Coding of Audiovisual Objects -Part 10: Advanced Video coding. 2003 and ITU-T Recommendation H.264 :Advanced video coding for generic audiovisual services.

2. Vetro, A.; Christopoulos, C.; Sun, H, "Video Transcoding Architectures and Techniques: An Overview”, IEEE Signal Processing Magazine, Vol.20, Issue2, pp.18-29, March 2003.

3. H. Sun, W. Kwok, and J. Zdepski, “ architectures for MPEG compressed bitstream scaling”, IEEE Trans. Circuits Syst. Video Technol., vol. 5, pp.191-199, April 1996.

4. N. Bjork and C. Christopoulos, "Trascoder architectures for video coding”, IEEE Trans. Consumer Electron., vol.44, pp.88-98, February 1998.

5. J. Youn, M.T. Sun, and C.W. Lin, "Motion vector refinement for high performance transcoding", IEEE Trans. Multimedia, vol.1, pp.30-40, March 1999.

6. J.N. Hwang, T.D. Wu, and C.W. Lin, "Dynamic frame-skipping in video transcoding", In Proc. IEEE Workshop Multimedia signal processing, Redondo Beach, CA, pp.616-621, December1998.

7. Kalva, H.; Vetro, A.; Sun, H., "Performance Optimization of the MPEG-2 to MPEG-4 Video Transcoder", SPIE Conference on VLSI Circuits and Systems, Vol. 5117, pp.341350, May 2003.

8. Haiyan Shu, "An Efficient Arbitrary Downsizing Algorithm for Video", Transcoding, IEEE Trans. Circuits Syst. Video Technol., vol.14, no.6, pp.887-891, June 2004.

9. Kwang-deok Seo, Jae-Kyoon Kim: "Fast motion vector re-estimation for transcoding MPEG-1 into MPEG-4 with lower spatial resolution in DCT-domain", Signal Processing: Image Communication, vol.19, pp.299-312, 2004.

10. Iain E.G. Richardson, H.264 and MPEG-4 video compression. Willey, 2003.

11. Henrique S. Malvar, "Low-Complexity Transform and Quantization in H.264/AVC", IEEE Trans. Circuits. Syst. Video Technol,. vol.13. no.7, pp.598-602, July 2003.

12. http://www.mpeg.org/MPEG/MSSG/tm5/

13. http://iphome.hhi.de/suehring/tml/download/ 